\title{
Praxis der parenteralen Ernährung
}

Die Applikation von Infusionslösungen zur parenteralen Ernährung kann über periphere Venen mit-tels Nadel, Braunüle oder kurzem Katheter erfol-gen oder über einen zentralvenösen Zugang (Vena jugularis interna und externa, Vena subclavia, Vena basilica). Die periphere Infusion ist nur bei kurzfri-stiger Dauer bis zu 3 Tagen indiziert. Vorausset-zung ist, daß die Osmolarität der verwendeten Infusionslösungen 1200 mOsm/1 nicht übersteigt. In diese,m Grenzbereich liegen 10\%ige Aminosäuren-lösungen ohne Kohlenhydrate, 5\%ige Aminosäu-renlösungen mit 10\% Kohlenhydraten und 20\%ige Kohlenhydratlösungen. Höher konzentrierte Lö-sungen müssen über einen zentralen Venenkathe-ter appliziert werden. Bei peripher-venöser Infusion ist folgendes zu beachten:

1. Möglichst großes Kaliber der punktierten Vene im Verhältnis zur Punktionskanüle.

2. Gefäßschonende Punktion.

3. Ruhigstellung der betroffenen Extremität.

4. Kurzer Katheter bei Punktion im Gelenkbereich.

5. Pflege der punktierten Vene sowie der Punk-tionsstelle (mit antiphlogistischen bzw. antibiotischen Maßnahmen).

6. Nach 24-48 Stunden Venenwechsel zur kontra-lateralen Seite.

7. Exakte Dosierung der Infusionslösung.

Bei einer Infusionsdauer von mehr als 3 Tagen, bei Verwendung von Infusionslösungen mit einer Osmolarität von mehr als $1200 \mathrm{mOsm} / 1$ oder mit stark abweichendem pH-Wert (z. B. Tris), bei bedroh-lichem Kreislaufzustand und zur Messung des zentralen Venendrucks ist ein zentralvenöser Zugang indiziert. Aufgrund der neueren Literatur ist von alien zentralen Zugangswegen die Punktion der Vena jugularis interna mit der kleinsten Komplika-tionsrate behaftet. Wie BURRI zeigen konnte, sind zahlreiche Komplikationen jedoch vom Zugangs-weg unabhängig und werden vielmehr durch das angewandte Kathetermodell (Abbildung 1) bzw.

mangelnder Einlegetechnik verursacht. Zu bevor-zugen ist ein röntgendichter, weicher Katheter aus Polyäthylen mit Silikonüberzug. Der Katheter soll-te keine Weichmacher freisetzen und einen großen Innendurchmesser besitzen. Die Punktionskanüle sollte entfernbar sein. Seine sichere Verbindung zwischen Katheter und Infusionsbesteck ist selbst-verständlich.

Tabelle I soil die Aufstellung eines geeigneten In-fusionsregimes in Abhängigkeit von der Schwere 
Bp: Weichmacher aus PVC

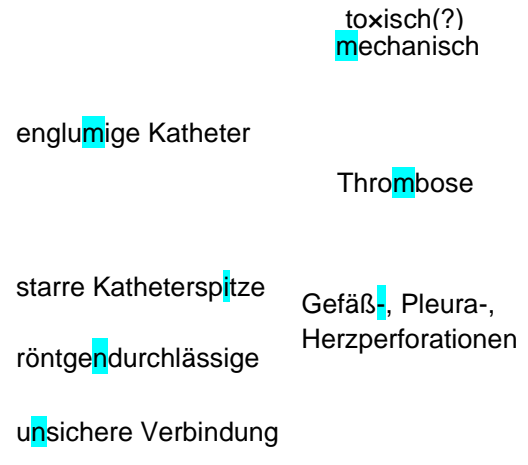

Luftembolie

nicht entfern bare Nadel

Katheterembolie

Abbildung 1. Verminderung der Kathetermaterial (Venoset $\left.{ }^{\circledR}\right)$.

der Katabolie und der Dauer der Bei kleinen operati-ven Eingriffen mit einer oralen Nahrungskarenz von 1-2 Tagen ist in der Regel ausschließliche Zu-fuhr von Wasser und Elektrolyten und einem Minimum an Kalorienträgern ausreichend. Bei mittel-schweren Eingriffen mit oraler Nahrungskarenz

Tabelle I. Differenzierte postoperative parenterale Substitution.

postoperativ pro Tag Operationstag

31 Tutofusin Op S 31 Tutofusin Op S

kleine Eingriffe mit oraler Nahrungskarenz von 1-2 Tagen

31 Tutofusin Op S 31TPE 1800

mittelschwere Eingriffe mit oraler

1,51AminufusinL600 1,5 Nahrungskarenz bis zu 4 Tagen
lTriofusinE 1000

31 Tutofusin Op S

große Eingriffe mit oraler Nahrungskarenz von mehr als 4 Tagen

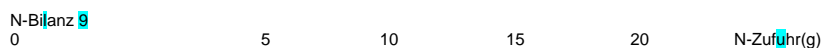

(nachịw.Hartịg)

Abbildung 2. Beziehungen zwischen Aminosäurenzufuhr, Grad der Katabolie und Stickstoffbilanz (nach Hartig).

bis zu 4 Tagen kann durch Applikation von 3000 ml TPE 1800 täglich eine fast ausgeglichene Stickstoffbilanz erzielt werden. Niedrigere Angebote an Aminosäuren und Kalorien stellten sich als unöko-nomisch heraus [Ahnefeld et al. 1976]. HARTIG fordert bei mittlerer Katabolie eine Mindeststickstoffzufuhr von ca. $11 \mathrm{~g}$ für eine einschneidende Verbesserung der Stickstoffbilanz \{Abbildung 2). Ein besonderer Vorteil von TPE 1800 liegt darin, daß es aufgrund seiner relativ niedrigen Osmolari-tät von $1100 \mathrm{mOsm} / 1$ und seines physiologischen $\mathrm{pH}$ von 7,4 auch für die Applikation in periphere Venen geeignet ist. Bei großen operativen Eingriffen mit oraler Nahrungskarenz von mehr als 4 Tagen ist eine komplette parenterale Ernährung über einen zentralvenösen Katheter erforderlich. Hierbei hat sich die Kombination von Aminofu-sion ${ }^{\circledR L} 600$ und Triofusion ${ }^{\circledR} E 1000$ bewährt.

\section{Literatur}


Ahnefeld, F. W. und Dölp, R.: Elektrolytthera-pie in der intra- und postoperativen Phase; in Beisbarth, Horatz und Rittmeyer Die Bau-steine der parenteralen Ernährung. Symposium am 21.10.1972 in Hamburg-Eppendorf (Enke, Stuttgart 1973).

Ahnefeld, F. W., Dölp, R. und Mehrkens, H.: Mög-lichkeiten der peripheren parenteralen Ernährung in der postoperativen Phase (vorläufige Mittei-lung). Infusionstherapie 3:195-197 (1976).

BURRI, C. und KRISCHAK, G.: Technik und Gefahren des Kava-Katheters. Infusionstherapie 3: 174179 (1976).

HARTIG, W.: Ernährung des Patienten im Stress. Zblı. Chir. 99:577-586(1974).

Pemsel, W. und GOFFERJE, H.: Klinische Erfahrungen mit TPE 1800. Bilanzierte Ernährung in der Therapie: II. Internationales Symposium, Erlangen, 17. bis 19.6.1976. 